\title{
ANÁLISIS DE LOS PRINCIPALES FACTORES DE RIESGO PREEXISTENTES EN PACIENTES DIAGNOSTICADOS CON COVID- 19 EN AMAPÁ, AMAZÔNIA, BRASIL
}

\section{ARTÍCULO ORIGINAL}

OLIVEIRA, Arthur Aires de ${ }^{1}$, MOREIRA, Danilo José Silva², PIMENTEL, Jhon Allyson Sena $^{3}$, COSTA, Pedro Henrique de Magalhães ${ }^{4}$, DIAS, Claudio Alberto Gellis de Mattos $^{5}$, ARAÚJO, Maria Helena Mendonça de ${ }^{6}$, OLIVEIRA, Euzébio de DENDASCK, Carla Viana ${ }^{8}$, SOUZA, Keulle Oliveira da $^{9}$, FECURY, Amanda Alves ${ }^{10}$

OLIVEIRA, Arthur Aires de. Et al. Análisis de los principales factores de riesgo preexistentes en pacientes diagnosticados con COVID-19 en Amapá, Amazônia, Brasil. Revista Científica Multidisciplinar Núcleo do Conhecimento. Año 06, Ed. 06, Vol. 17, pp. 56 y 72. Junio de 2021. ISSN: 2448-0959, Enlace de acceso: https://www.nucleodoconhecimento.com.br/salud/riesgo-preexistente, DOI: 10.32749/nucleodoconhecimento.com.br/salud/riesgo-preexistente

\footnotetext{
${ }^{1}$ Un estudioso de la medicina. Universidad Federal de Amapá (UNIFAP).

${ }^{2}$ Un estudioso de la medicina. Universidad Federal de Amapá (UNIFAP).

${ }^{3}$ Un estudioso de la medicina. Universidad Federal de Amapá (UNIFAP).

${ }^{4}$ Un estudioso de la medicina. Universidad Federal de Amapá (UNIFAP).

${ }^{5}$ Biólogo, Doctor en Teoría e Investigación del Comportamiento, Profesor e investigador del Curso de Grado de Química del Instituto de Educación Básica, Técnica y Tecnológica de Amapá (IFAP) y del Programa de Posgrado en Educación Profesional y Tecnológica (PROFEPT IFAP).

${ }^{6}$ Doctor, Máster en Docencia y Ciencias de la Salud, Profesor e investigador del Curso Médico del Campus Macapá, Universidad Federal de Amapá (UNIFAP).

${ }^{7}$ Biólogo, Doctor en Enfermedades Tropicales, Profesor e investigador del Curso de Educación Física de la Universidad Federal de Pará (UFPA).

${ }^{8}$ Teóloga, Doctora en Psicoanálisis Clínica. Trabaja desde hace 15 años con Metodología Científica (Método de Investigación) en Orientación de Producción Científica para Estudiantes de Maestría y Doctorado. Especialista en Investigación de Mercados e Investigación en Salud, Estudiante de Doctorado en Comunicación y Semiótica (PUC SP).

${ }^{9}$ Científico Social, Especialista en Gestión y Docencia de la Educación Superior, Estudiante de Maestría en Estudios Antrópicos en la Amazonía (UFPA).

${ }^{10}$ Biomédico, Doctor en Enfermedades Tropicales, Profesor e investigador del Curso Médico del Campus Macapá, Universidad Federal de Amapá (UNIFAP), Pro-Rector de Investigación y Estudios de Posgrado (PROPESPG) de la Universidad Federal de Amapá (UNIFAP).
}

RC: 90561

Enlace de acceso: https://www.nucleodoconhecimento.com.br/salud/riesgo-preexistente 


\section{RESUMEN}

EI COVID-19 (Coronavirus Disease 2019) es una infección causada por el virus SARSCoV-2, con los primeros casos reportados en diciembre de 2019 en la ciudad de Wuhan. Se encontró que en pacientes con comorbilidades, existe un mayor riesgo de complicaciones y mortalidad en caso de infección. La enfermedad fue considerada por la Organización Mundial de la Salud en 2020 como una emergencia de salud pública y, en Brasil, el Ministerio de Salud implementó medidas para el monitoreo epidemiológico de los casos registrados en las diferentes unidades federativas del país. Entre estos, Amapá asume altos niveles de incidencia y mortalidad. Este estudio tiene como objetivo analizar los principales factores de riesgo previos encontrados en pacientes con COVID-19 en el Estado de Amapá. Para ello, se utilizaron datos secundarios disponibles en el Panel Coronavirus Amapá a través de una búsqueda realizada el 22 de mayo de 2020. En la plataforma se recogieron datos sobre el número de pacientes diagnosticados con COVID-19, así como el número de muertes por la enfermedad, con evaluación conjunta en cada tema de comorbilidades encontradas. Después de agrupar estos datos en hojas de cálculo de Microsoft Excel, se realizó un análisis cuantitativo y descriptivo de estos. En el Estado, un total de 28,927 pacientes diagnosticados con COVID-19 fueron registrados hasta el día de la recolección. Alrededor del 1,31\% (378 casos) de estos tenían un factor de riesgo que podría susceptibles a desarrollar complicaciones derivadas del COVID-19, siendo la diabetes y la cardiopatía crónica las afecciones con mayor número de registros. Aún en el Estado de Amapá se encontraron 420 muertes en pacientes con COVID-19. De este total, el 75,71\% (318 casos) tenía factores de riesgo previos de mayor riesgo de complicaciones por COVID-19, siendo la hipertensión y la diabetes las condiciones predominantes. Con el estudio, se observó en Amapá que gran parte de la población de pacientes diagnosticados con la enfermedad y fallecidos tenían antecedentes de factor de riesgo por COVID-19. Debido a la notificación de factores de riesgo en la situación de muerte que no se registraron en el número de pacientes diagnosticados, así como la inespecífica inherente a la condición de los pacientes monitoreados por los mecanismos de salud del estado, no fue posible realizar un análisis más detallado 
sobre el grado de complicación y la tasa de letalidad generada por un determinado factor de riesgo y su asociación con la infección por coronavirus.

Palabras clave: COVID-19, Factores de riesgo, Comorbilidad.

\section{INTRODUCCIÓN}

En la primera quincena de diciembre de 2019 , se había reportado el primer caso de neumonía de origen desconocido en Wuhan, provincia de Hubei, China. Posteriormente, un brote de neumonía por síndrome respiratorio agudo severo (SRAG) asoló la provincia, llamando la atención de la Organización Mundial de la Salud (OMS), que, partiendo de la circunscripción de sus atribuciones, declaró que la situación era una epidemia causada por un nuevo subtipo de virus perteneciente a la familia coronaviridae, el coronavirus-2, que constituye una Emergencia de Salud Pública de Importancia Internacional (ESPII), el 30 de enero de 2020 (COSTA et al., 2020a; FERRARI, 2020; GAUTRET et al., 2020; GUAN et al., 2020; LI et al., 2020; LIMA et al., 2020; MARTINS-CHAVES; GOMES; GOMEZ, 2020; OLIVEIRA et al., 2020; RENU; PRASANNA; GOPALAKRISHNAN, 2020; RODRÍGUEZ-COLA et al., 2020; TAY et al., 2020; ZHAl et al., 2020).

En febrero de 2020, el Director General de la OMS nombró a la enfermedad coronavirus-19 (COVID-19), una infección causada por el SARS-CoV-2 (de la expresión inglesa: Severe Acute Respiratory Syndrome Coronavirus 2), uno de los siete subtipos de coronavirus conocidos (COSTA et al., 2020a; RENU; PRASANNA; GOPALAKRISHNAN, 2020; HONG et al., 2020).

Desde la perspectiva brasileña, la infección por COVID-19 fue declarada por el Ministerio de Salud (EM) como Emergencia de Salud Pública de Importancia Nacional (ESPIN) el 3 de febrero de 2020, y su primer caso fue confirmado en Brasil el 26 de febrero (OLIVEIRA et al., 2020; OLIVEIRA; LUCAS; IQUIAPAZA, 2020). Como medida de apoyo al Sistema de Salud, el Departamento de Informática del Sistema Único de Salud (DATASUS), a través del Plan de Contingencia, actuó brindando asistencia virtual a la población a través de la aplicación "Coronavírus SUS" (BRASIL, 2020a). 
En Brasil, el progreso de la pandemia es monitoreado principalmente por el MS a través de índices estadísticos que reflejan el estado de cada unidad federativa (UF), y el Estado de Amapá asume altos índices epidemiológicos, especialmente en lo que respecta al coeficiente de incidencia de COVID-19 (BRASIL, 2020c).

Hasta el 17 de julio de 2020, Amapá lideró el Norte brasileño en el ranking del Coeficiente de Incidencia COVID-19 por UF notificador (número de casos confirmados por cada 100.000 habitantes), cuyo valor fue de $3.637,4$ seguido de Roraima, con 3.327,1, que a su vez fue subsecuenciado por el Estado de Amazonas, con 1962,0. En cuanto al Coeficiente de Mortalidad COVID-19 (número de muertes por cada 100.000 habitantes), Amapá quedó en el $7^{\circ}$ lugar $(55,2)$ (BRASIL, 2020c).

Los factores de riesgo se relacionan con cualquier condición que afecte la respuesta inmune del cuerpo a una enfermedad. Así, estos factores incluyen tanto las comorbilidades, es decir, las enfermedades crónicas previamente instaladas y que actúan concomitantemente en el organismo del paciente, así como las particularidades de los individuos, a saber: condición puerperal, tabaquismo, consumo de alcohol, entre otros (PIRES; ROBLE; XAVIER, 2020; ENGIN; ENGIN; ENGIN, 2020; KHALIQ; PHOSWA, 2020).

Los estudios sugieren que los pacientes infectados con COVID-19 que tienen comorbilidades tienen una mayor vulnerabilidad inmunológica, así como una mayor tasa de letalidad en comparación con los pacientes afectados solo por el virus. Las principales comorbilidades enumeradas incluyen hipertensión, diabetes, enfermedades cardíacas y enfermedades crónicas del sistema respiratorio (COSTA et al., 2020a; FERRARI, 2020; MARTINS-CHAVES; GOMES; GOMEZ, 2020; RENU; PRASANNA; GOPALAKRISHNAN, 2020; YANG et al., 2020).

Dado el panorama global y en vista del estado del Estado de Amapá, es esencial realizar análisis, estudios y promociones en la investigación científica, con el fin de establecer manejos adecuados para los pacientes afectados por enfermedades subyacentes y/o condiciones que representen potenciales factores de riesgo para el pronóstico de los pacientes diagnosticados con SARS-CoV-2, con el objetivo de 
mitigar las complicaciones presentadas por ellos (RENU; PRASANNA; GOPALAKRISHNAN, 2020; YANG et al., 2020).

\section{METAS}

Analizar la distribución de los principales factores de riesgo preexistentes en pacientes diagnosticados de COVID-19 en el Estado de Amapá, Amazônia, Brasil.

\section{METODOLOGÍA}

Este estudio tuvo como objetivo realizar un análisis cuantitativo y descriptivo del número de pacientes afectados por COVID-19 en Amapá que dan fe de factores de riesgo para la enfermedad, y para ello, se realizó una consulta el 22 de mayo de 2020 en el Panel Coronavirus Amapá (painel.corona.ap.gov.br).

En la página principal del Panel Coronavirus Amapá, se hizo clic en "descargar CSV" para obtener los principales datos de casos de covid-19 en el Estado de Amapá. Luego, en las "Comorbilidades de pacientes confirmados" de la misma página, se seleccionó la opción "Inspect" en la esquina superior derecha para generar una lista de comorbilidades y su relación numérica. El mismo proceso se repitió en las "Comorbilidades de pacientes moribundos". Posteriormente, para cada una de las listas obtenidas, se seleccionó la opción "Fromatted CSV" en el campo "Download CSV" para obtener los archivos y utilizarlos en Microsoft Excel.

Dentro de estos ficheros, algunas comorbilidades y situaciones de riesgo se unieron en el mismo conjunto con el fin de estandarizar los resultados. A ser: se agrupan -en Enfermedades Cardíacas Crónicas- cardiopatías crónicas, cardiopatías crónicas, cardiopatías crónicas, cardiopatías crónicas, enfermedades cardiovasculares, cardiopatías, cardiopatías, cardiopatías crónicas, cardiopatías; también están en Enfermedades Respiratorias Crónicas - enfermedades respiratorias crónicas, enfermedades respiratorias crónicas, enfermedades respiratorias crónicas descompensadas crónicas; Todavía están en - Hipertensión- hipertensión, HAS, hipertenso; otra vez están en - Enfermedad cardíaca e hipertensión- Enfermedad 
cardíaca crónica (HAS), enfermedad cardíaca crónica - HAS, enfermedad cardiovascular incluyendo hipertensión, enfermedad cardíaca crónica HAS, enfermedad cardíaca (HAS), enfermedad cardíaca crónica - HAS; así como en Inmunosupresión- inmunosupresión, portador de la enfermedad cromosómica o estado de FR; Otra vez están en - Enfermedades del corazón y diabetes enfermedades cardíacas crónicas y diabetes, corazón y diabéticos; Por lo tanto, están en un embarazo de alto riesgo: mujer embarazada de alto riesgo, mujer embarazada de alto riesgo; Finalmente están en - Enfermedades renales crónicas en etapa avanzada - Enfermedad renal crónica en etapa avanzada, Enfermedad renal crónica en etapa avanzada, Enfermedad renal crónica en etapa avanzada.

En la hoja de cálculo "Comorbilidades de pacientes en muerte", el número de casos registrados como "Sin información" fueron ignorados debido a la inconclusividad de este corte. Los demás datos fueron seleccionados y agrupados como se describió anteriormente.

El presente estudio utilizó datos secundarios disponibles en el dominio público y tuvo en cuenta aspectos éticos basados en la Resolución 466/12.

\section{RESULTADOS}

Entre los 28.927 casos confirmados de COVID-19 en Amapá que fueron reportados hasta el día de la recolección, aproximadamente 1,31\% de los pacientes (378 casos) tenían un factor de riesgo que podría susceptibles de desarrollar complicaciones debido a la afectación del SARS-CoV-2, como se muestra en la Figura 1. 
Figura 1 - Presencia de factores de riesgo en pacientes diagnosticados de COVID-19 en Amapá hasta el 22 de mayo de 2020.

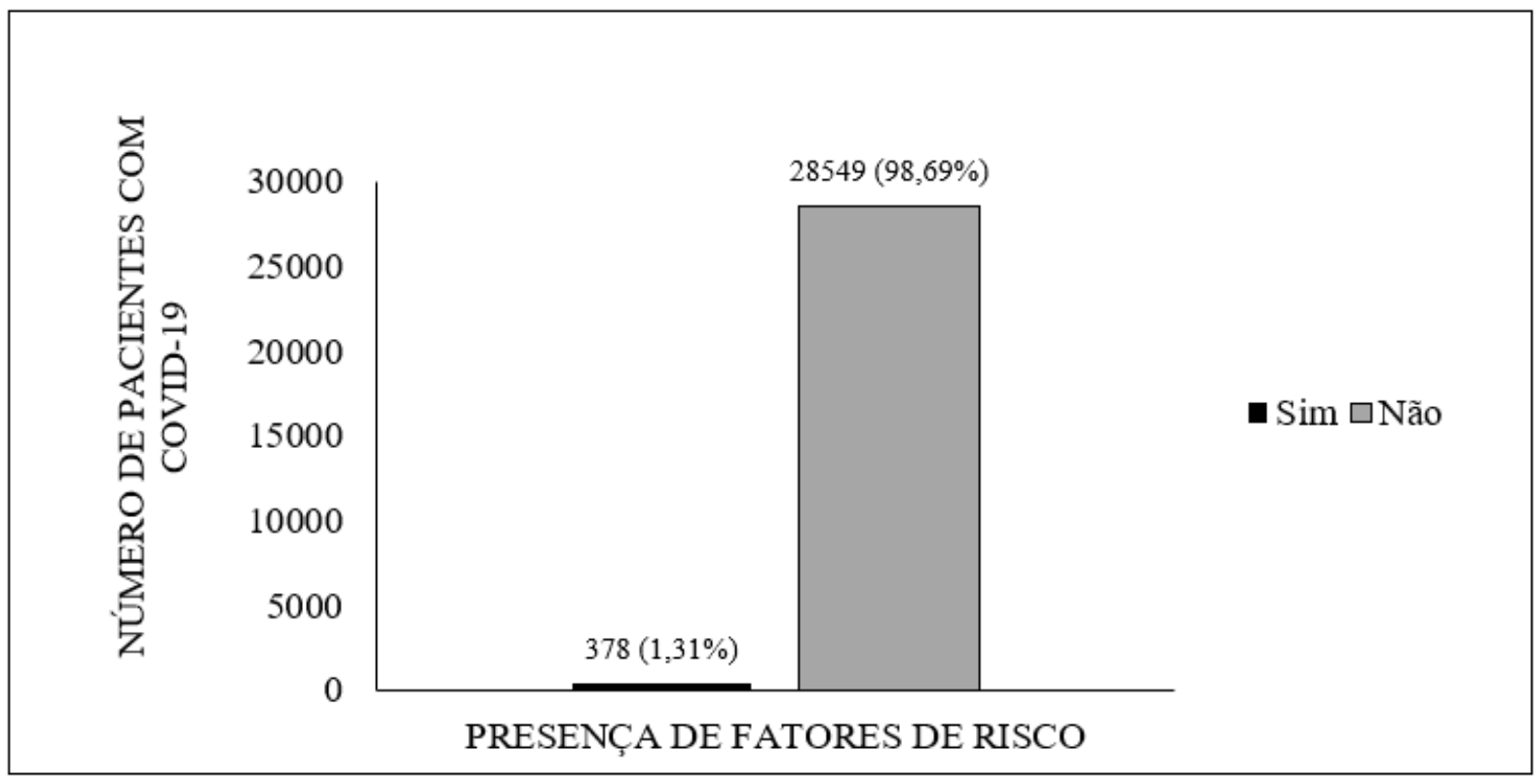

Fuente: Panel Coronavirus Amapá.

En cuanto a los pacientes que tenían factores de riesgo, el 5,02\% de ellos (19 casos) tenían más de uno. Las asociaciones incluyen enfermedad cardíaca e hipertensión $(3,44 \%)$, enfermedad cardíaca y diabetes $(1,06 \%)$, angioplastia y ateromatosis coronaria $(0,26 \%)$ y enfermedad renal crónica y diabetes $(0,26 \%)$.

En cuanto a los principales factores de riesgo relatados solos por los pacientes afectados, se encontró que la mayoría tenía diabetes 29,89\% (112 casos), seguido de cardiopatías crónicas con $28,84 \%$ (109 casos).

Se observa que las enfermedades del ámbito cardiológico están presentes de manera mayoritaria, considerando que 173 pacientes en total están afectados por enfermedades de esta clase, sumando individuos con múltiples factores de riesgo e individuos solo con cardiopatía. 
Tabla 1 - Distribución de factores de riesgo en pacientes diagnosticados de COVID19 en Amapá.

\begin{tabular}{|c|c|c|}
\hline Factores de riesgo & $\begin{array}{l}\text { Número de } \\
\text { informes }\end{array}$ & Porcentaje(\%) \\
\hline Diabetes & 113 & $29,89 \%$ \\
\hline Enfermedad cardíaca crónica & 109 & $28,84 \%$ \\
\hline Enfermedades respiratorias crónicas & 71 & $18,78 \%$ \\
\hline hipertensión & 46 & $12,17 \%$ \\
\hline Enfermedades del corazón e hipertensión & 13 & $3,44 \%$ \\
\hline inmunosupresión & 11 & $2,91 \%$ \\
\hline Enfermedades del corazón y diabetes & 4 & $1,06 \%$ \\
\hline Embarazo de alto riesgo & 4 & $1,06 \%$ \\
\hline $\begin{array}{l}\text { Enfermedad crónica crónica en estadio } \\
\text { avanzado }\end{array}$ & 3 & $0,79 \%$ \\
\hline Angioplastia y Ateromatosis Coronaria & 1 & $0,26 \%$ \\
\hline $\begin{array}{l}\text { Enfermedades cardíacas crónicas y } \\
\text { diabetes }\end{array}$ & 1 & $0,26 \%$ \\
\hline Cirrosis hepática & 1 & $0,26 \%$ \\
\hline depresión & 1 & $0,26 \%$ \\
\hline Total & 378 & $100,00 \%$ \\
\hline
\end{tabular}

Fuente: Panel Coronavirus Amapá.

En total, se registraron 420 muertes en el momento del estudio. De esta cuantitativa, se encontró que 318 pacientes tenían antecedentes de comorbilidad o predisponentes a complicaciones. 
Figura 2 - Presencia de factores de riesgo en pacientes fallecidos con COVID-19 en Amapá hasta el 22 de mayo de 2020.

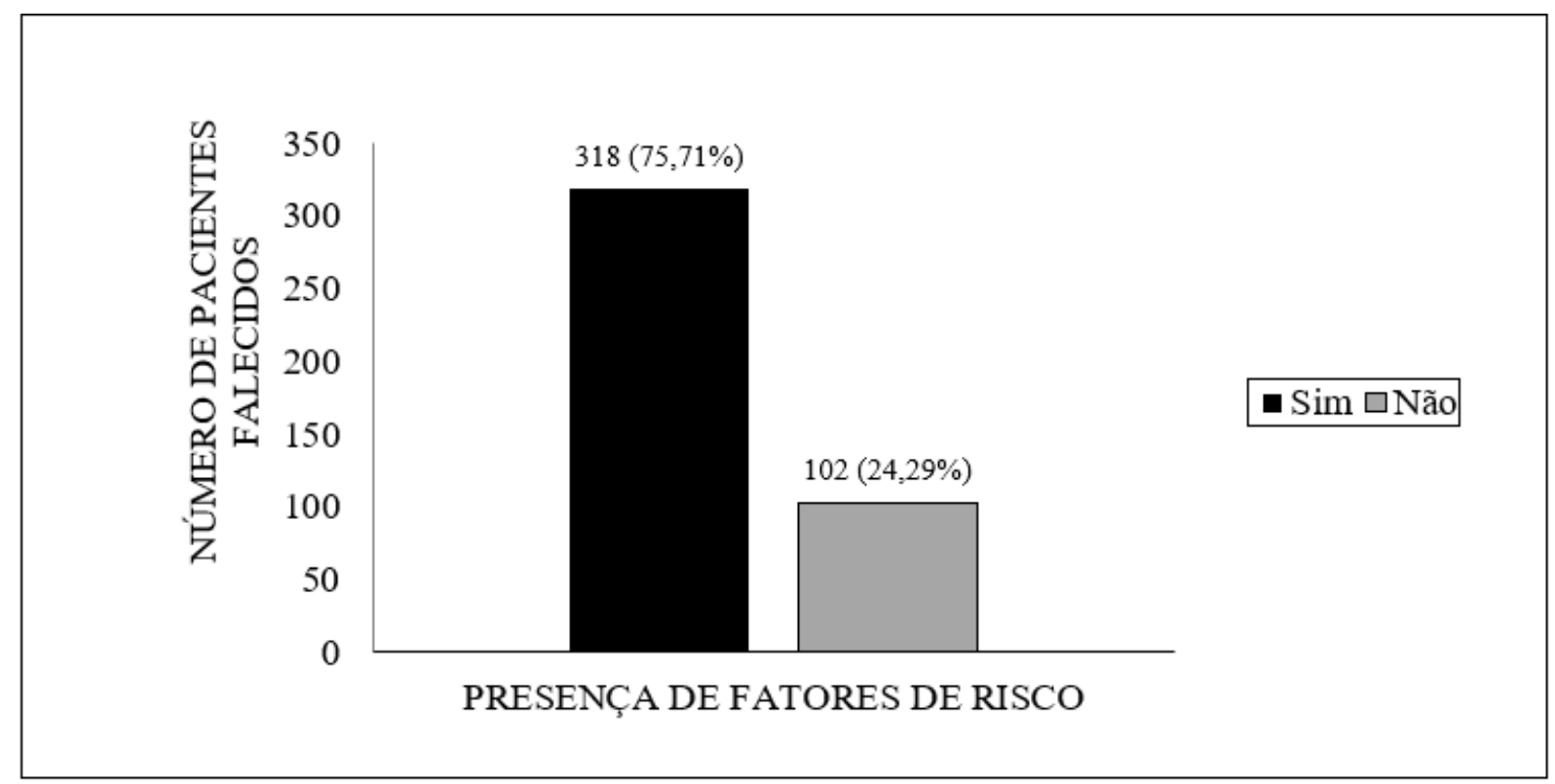

Fuente: Panel Coronavirus Amapá.

En cuanto a los factores de riesgo presentes en los pacientes fallecidos, el principal registro fue la hipertensión arterial, presente en el 38,68\% de los pacientes (123 casos). También se observó la presencia de otros factores agravantes que no fueron descritos previamente en individuos con infección confirmada. Son obesidad, enfermedad cerebrovascular, neoplasia, tabaquismo, Alzheimer, enfermedad del páncreas, dengue, consumo de alcohol y puerperal. 
Tabla 2 - Distribución de los factores de riesgo registrados en pacientes fallecidos con COVID-19 en Amapá.

\begin{tabular}{|l|c|c|}
\hline Comorbilidad & Número de muertes & Porcentaje (\%) \\
\hline hipertensión & 123 & $38,68 \%$ \\
\hline Diabetes & 93 & $29,25 \%$ \\
\hline Enfermedades de la piel & 27 & $8,49 \%$ \\
\hline corazón & 19 & $5,97 \%$ \\
\hline obesidad & 19 & $5,97 \%$ \\
\hline Enfermedades respiratorias & 15 & $4,72 \%$ \\
\hline Enfermedades cerebrovasculares & 8 & $2,52 \%$ \\
\hline Enfermedades inmunosupresoras & 3 & $0,94 \%$ \\
\hline neoplasmas & 3 & $0,94 \%$ \\
\hline tabaquismo & 2 & $0,63 \%$ \\
\hline Alzheimer & 1 & $0,31 \%$ \\
\hline Enfermedad del páncreas & 1 & $0,31 \%$ \\
\hline Enfermedad hepática & 1 & $0,31 \%$ \\
\hline dengue & 1 & $0,31 \%$ \\
\hline Etilista & 1 & $0,31 \%$ \\
\hline Puerpera & 1 & $0,31 \%$ \\
\hline Total & 318 & $100,00 \%$ \\
\hline
\end{tabular}

Fonte: Painel Coronavírus Amapá.

\section{DISCUSIÓN}

Los estados de la región amazónica de Brasil tienen considerables discrepancias socioeconómicas en comparación con los demás, situación que puede reflejar el mayor impacto en el sistema de salud observado. En el Estado de Amapá, tras la denuncia del primer caso el 25 de marzo de 2020, el COVID-19 se propagó masivamente, así como en las demás unidades federativas (DIAS et al., 2020; MENDONÇA et al., 2020). 
El número de pacientes con COVID-19 que tienen factores de riesgo para la enfermedad en Amapá, en comparación con el número total de pacientes infectados, se considera bajo. Existe consenso en la literatura en que las comorbilidades y situaciones de vulnerabilidad inmunológica se consideran factores agravantes para la condición de COVID-19, y este hecho puede haber llevado a los individuos de los grupos de riesgo a redoblar su atención (COSTA et al., 2020b; RODRÍGUEZ-COLA et al., 2020).

En un estudio realizado por Wang et al. (2020), se informó que de 36 pacientes que requirieron atención en la Unidad de Cuidados Intensivos (UTI), 26 (72,2\%) tuvo comorbilidades, un hallazgo que indica que una historia de enfermedades crónicas puede conducir al desarrollo de complicaciones. Silva et al. (2020), en un estudio de caracterización clínica y epidemiológica con 1560 pacientes diagnosticados de COVID-19 de la ciudad de Macapá-AP, encontró que 102 pacientes (6,54\%) comorbilidades.

Las principales comorbilidades registradas solas en los pacientes del presente estudio fueron diabetes, cardiopatías crónicas, enfermedades respiratorias crónicas e hipertensión arterial. Un metanálisis realizado con un total de 1576 participantes por Yang et al. (2020) también destaca la hipertensión $(21,1 \%)$, la diabetes $(9,7 \%)$, las enfermedades cardiovasculares $(8,4 \%)$ y enfermedades del sistema respiratorio $(1,5 \%)$ como las comorbilidades más frecuentes. Silva et al. (2020) encontró que de los 102 pacientes con comorbilidades diagnosticadas con COVID-19 evaluadas, enfermedades cardiovasculares crónicas (38,33\%), diabetes $(24,16 \%)$ y enfermedades respiratorias crónicas $(13,33 \%)$ fueron los factores de riesgo más prevalentes. Estas condiciones están asociadas con un alto riesgo de manifestaciones graves de COVID-19 (GOMES; GÓMEZ, 2020; MARTINS-CHAVES), que se explica en partes por el aumento de la expresión de la enzima convertidora de angiotensina 2 (ACE2) en células epiteliales alveolares, cardíacas, $\beta$ pancreáticas y vasculares del endotelio. ECA2 permite la entrada del virus en las células del cuerpo, un hecho que corrobora la falla de los órganos correspondientes durante la condición infecciosa (ANGHEBEM; REGO; TARTH, 2020; ASKIN; TANRIVERDI; ASKIN, 2020). 
Algunos pacientes también tenían otras comorbilidades y factores de riesgo, incluyendo inmunosupresión, embarazo de alto riesgo, enfermedades renales crónicas, angioplastia con ateromatosis coronaria, cirrosis hepática y depresión.

Los datos del presente estudio muestran una letalidad para los pacientes con enfermedades inmunosupresoras del $27,27 \%$. Se cree que cuanto más comprometida es la inmunidad de los pacientes con COVID-19, peor es el pronóstico de la enfermedad, razón que enmarca a los pacientes inmunodeprimidos como grupos de riesgo de la enfermedad (GOMES; GÓMEZ, 2020; MARTINS-CHAVES). Sin embargo, hay un informe en la literatura que la mayor parte de los pacientes immunosuppressed evaluados siguieron con un buen pronóstico. Un estudio realizado por Hrusak et al. (2020) con pacientes pediátricos sometidos a tratamiento anticancerígeno mostraron que de 9 niños diagnosticados con COVID-19, 8 seguidos con síntomas leves o incluso eran asintomáticos. Además, Bussalino et al. (2020) afirman que el mantenimiento de la inmunosupresión puede hacer que el curso de COVID-19 sea leve para el paciente al reducir la tormenta de citoquinas características de la enfermedad.

En cuanto al embarazo, se notificaron 4 casos de embarazo de alto riesgo en el Estado. Los riesgos maternales se sospechan porque los cambios inmunológicos sistémicos y locales se accionan en el cuerpo de la madre, extendiéndose de un estado favorable-inflamatorio a un estado antiinflamatorio (LIU y otros, 2020). En el caso de las mujeres embarazadas con COVID-19, un metanálisis realizado por Trippela et al. (2020) con 275 gestantes afectadas por la enfermedad mostraron que 269 describieron su sintomatología, y $91,82 \%$ presentaron síntomas, incluyendo fiebre y tos como los más frecuentes, y $8,18 \%$ eran asintomáticos. La mayoría de los pacientes presentan síntomas leves 0 asintomáticos, con pocos informes sobre el desarrollo de complicaciones (CAPARROS-GONZALEZ, 2020; TRIPPELA et al., 2020).

En relación a las enfermedades coronarias crónicas, solo se reportaron 3 casos confirmados al sistema de vigilancia epidemiológica del coronavirus amapá. Sin embargo, 27 casos de muertes de pacientes que previamente tenían comorbilidades 
de esta clase de enfermedades fueron insertados en el sistema, lo que sugiere un posible subregistro de comorbilidades. En un estudio de cohorte prospectivo producido por Cheng et al. (2020) con 701 individuos afectados por COVID-19, se observó que el $26,7 \%$ de las personas analizadas tenían hematuria y el $43,9 \%$ proteinuria. Estos hallazgos clínicos sugieren la presencia de problemas de laboratorio (MOITINHO et al., 2020). Dado que el SARS-CoV-2 actúa directamente sobre el receptor ECA2 (ASKIN; TANRIVERDI; ASKIN, 2020) y que ya se ha evidenciado una sobreexpresión de esta enzima en las células tubulares proximales de pacientes con enfermedades orales crónicas (ANA et al., 2020), se cree que la historia de enfermedades de la vida puede agravar el cuadro de COVID-19 (RENU; PRASANNA; GOPALAKRISHNAN, 2020). Además, se ha asociado a la preexistencia de enfermedades renales con el deterioro de la funcionalidad del sistema inmune del paciente, un proceso que podría explicarse por el proceso inflamatorio establecido durante la nefropatía. Esto fomenta la idea de que estas enfermedades son factores de riesgo para COVID-19 (OYELADE; ALQAHTANI; CANCIANI, 2020).

En cuanto a la relación entre la angioplastia y la ateromatosis coronaria con COVID19, no se encontraron informes en la literatura que pudieran apoyar la asociación entre estos factores de riesgo y la infección por el virus.

De los casos reportados de enfermedades hepáticas en el Estado de Amapá, sólo se ha reportado 1 caso de cirrosis hepática. También se registró la muerte de un paciente que tenía enfermedad hepática previa, pero no es posible afirmar que se trata del mismo paciente ya que no se especificó la enfermedad preexistente. La literatura muestra que la infección por SARS-CoV-2 también afecta al hígado (AGHAGOLI et al., 2020), y la presencia de enfermedades hepáticas se considera un factor determinante para la gravedad del cuadro de COVID-19 (RENU; PRASANNA; GOPALAKRISHNAN, 2020). Un metanálisis de Oyelade, Alqahtani y Canciani (2020) en el que participaron 5.595 pacientes mostró una prevalencia de enfermedades hepáticas en un paciente con COVID-19 del 3\%, y de estas, la gravedad se reportó en el $57,33 \%$. El mismo estudio reveló una tasa de mortalidad del $17,65 \%$. A pesar de 
la gravedad, no hubo correlación en la literatura entre la enfermedad hepática preexistente y el resultado de COVID-19.

En el único caso de depresión registrado en el Estado, existe una preocupación de la comunidad científica sobre la posibilidad de que las enfermedades neuropsíquicos también constituyan un riesgo para la condición de COVID-19. Los trastornos de salud mental, así como el estado de angustia psicológica, influyen directamente en el funcionamiento del sistema inmunológico de un individuo, que puede susceptible a infecciones virales de las vías respiratorias, lo que evidencia una posible relación entre el estado emocional del paciente y el grado de afectación del SARS-CoV-2 (RAJKUMAR, 2020). Sin embargo, los efectos generados por el virus en los pacientes en cuestión, así como la respuesta inmunológica generada por el organismo, no están bien dilucidados en la literatura (TROYER; KOHN; HONG, 2020). Para los pacientes con antecedentes de debilidad en la salud mental, se debe prestar mayor atención a la afección, ya que puede recaer o empeorar en medio del escenario de pandemia instalado y un posible diagnóstico de COVID-19 (YAO; CHEN; XU, 2020).

Los datos recogidos del panel de coronavirus de Amapá indican que el $76 \%$ de los pacientes fallecidos tenían comorbilidades o agravantes preexistentes. An epidemiological survey by Almeida et al. (2020) en el Estado de Maranhão encontró que de los 100 pacientes diagnosticados de COVID-19 y que murieron, $78 \%$ tenían comorbilidades previas, asumiendo un patrón similar al observado en Amapá.

Se observa que la Hipertensión arterial es el factor de riesgo más registrado entre los pacientes amapaenses fallecidos. Sin embargo, no es posible determinar la tasa de letalidad para los pacientes hipertensos, ya que el número de casos confirmados de la enfermedad es menor que el reportado de muerte. Sin embargo, la literatura muestra que esta enfermedad es uno de los principales agravantes de la enfermedad COVID-19, junto con otras enfermedades cardiovasculares (AGHAGOLI et al., 2020; ALMEIDA et al., 2020; FERRARI, 2020). El mecanismo fisiopatológico propuesto para explicar esta relación implica el sistema reinthine. Debido a la mayor unión del SARSCoV-2 a ECA2, la concentración de esta enzima se reduce en el huésped, lo que resulta en altos niveles de angiotensina II y disminución de la angiotensina 1-7, efectos 
que generan vasoconstricción y retención de sodio y empeoran la condición hipertensiva (TADIC et al., 2020).

En cuanto a la relación entre diabetes y COVID-19, es posible empeorar las condiciones clínicas del paciente, considerando que el SARS-COV-2 promueve el aumento de la glucosa en sangre en pacientes con diabetes tipo 2 (BORNSTEIN et al., 2020). El empeoramiento de la condición de los diabéticos puede estar relacionado con el hecho de que esta comorbilidad disminuye la inmunidad natural del cuerpo (COSTA et al., 2020b; MA; HOLT, 2020). En relación a los casos reportados de esta enfermedad en Amapá, se nota que el número de pacientes confirmados (112) es cuantitativamente cercano a los fallecidos (93), corroborando así la idea de que la diabetes puede agravar la condición de covid-19 hasta el punto de que el paciente fallece (COSTA et al., 2020b; RONCON et al., 2020).

Otra característica importante de la relación diabetes-COV-2 son los mecanismos de complicaciones resultantes de la unión de estos dos factores, ya que los individuos que tienen diabetes y están infectados por un virus tienen más riesgo de desarrollar complicaciones (LACOBELLIS, 2020). Una hipótesis sobre la diabetes que actúa negativamente, junto con el virus, está relacionada con ECA2, ya que está presente en varios tejidos y actúa como una puerta de entrada a agentes infecciosos en la superficie celular (AGHAGOLI et al., 2020; MA; HOLT, 2020). Además, los pacientes que usan inhibidores enzimáticos supresores de angiotensina (IECA) son más propensos a desarrollar complicaciones debido a una mayor producción de ECA2, lo que favorece la acción del SARS-CoV-2 (FANG; KARAKIULAKIS; ROTH, 2020; FERRARI, 2020; MA; HOLT, 2020).

Las enfermedades cardíacas, a su vez, son condiciones asociadas con peores pronósticos y altas tasas de letalidad (AGHAGOLI et al, 2020). Un metanálisis de Huang, Wang y Li (2020) encontró que de los 41 pacientes con COVID-19 evaluados, $6(15 \%)$ tenía enfermedades de la esfera cardíaca. De hecho, hay un número considerable de pacientes con cardiopatías que murieron en Amapá (19), generando una tasa de letalidad de $15,07 \%$. La literatura asocia esta gravedad con la presencia de receptores ECA2 en el corazón, que están altamente expresados en el órgano, lo 
que puede conducir al desarrollo de lesión miocárdica aguda y daño cardiovascular crónico (AGHAGOLI et al, 2020). Además, el efecto de edad avanzada e imunosenescencia observado en la mayoría de los pacientes con enfermedad cardíaca puede estar asociado con el pronóstico de la enfermedad COVID-19 (DRIGGIN et al., 2020).

También se observó un número considerable de pacientes con enfermedades respiratorias, lo que corresponde a una tasa de letalidad del $21,12 \%$ teniendo en cuenta el número de pacientes diagnosticados de COVID-19 que reportaron comorbilidades preexistentes. La preexistencia de enfermedades respiratorias se asocia con condiciones graves de COVID-19 (MARTINS-CHAVE; GOMES; GOMEZ, 2020; YANG et al., 2020). Se sabe que el sistema inmune responde a la Infección por SARS-CoV-2 con un proceso inflamatorio marcado por la acción de las células inmunes, necrosis tisular e hiperplasia local, efectos que pueden perjudicar el proceso de intercambio gaseoso de los pulmones y generar un cuadro de neumonía grave (GIMENEZ et al., 2020), lo que puede justificar por qué las enfermedades respiratorias son un factor de riesgo para el COVID-19.

También en la información proporcionada por el Panel Coronavirus Amapá, se observa que algunos factores de riesgo por COVID-19 encontrados en pacientes fallecidos no fueron reportados de la sesión de factores de riesgo de pacientes diagnosticados con la enfermedad, tales como obesidad, enfermedades cerebrovasculares, neoplasias, tabaquismo, enfermedad de Alzheimer, enfermedad pancreática, dengue, alcoholismo y puerperal. Esto impide un análisis fiable del escenario epidemiológico de la localidad. Sin embargo, algunos aspectos sobre estas condiciones se han discutido en la literatura.

Sobre la obesidad, se ha sabido durante mucho tiempo que se asocia directamente con pronósticos pobres y una recuperación lenta en casos de infecciones virales. Este hallazgo se atribuye al estado inflamatorio constante característico de los individuos obesos, apoyado por altos niveles de fármacos inflamatorios, lo que contribuye al deterioro de la respuesta inmunológica generada por estos pacientes $\mathrm{y}$, en consecuencia, a la disminución de la capacidad de combatir agentes infecciosos 
(COSTA et al., 2020b; HUSSAIN et al., 2020; KORAKAS et al., 2020). En el contexto de COVID-19, otros factores asociados con la obesidad pueden ser responsables de las mayores posibilidades de empeoramiento del cuadro clínico, que incluyen mecánica respiratoria deficiente, baja tasa de intercambio de gases en los pulmones, aumento de la resistencia de las vías respiratorias, reducción de la fuerza pulmonar y menor volumen pulmonar (STEFAN et al., 2020). Un metanálisis realizado por Hussain et al. (2020) mostró que de los 2.451 pacientes con Índice de Masa Corporal (IMC) superior a $25 \mathrm{~kg} / \mathrm{m} 2$ analizados, 531 fallecieron, lo que corresponde a una tasa de letalidad del 21,66\%. El mismo estudio también evaluó pacientes con IMC inferior a $25 \mathrm{~kg} / \mathrm{m}^{2}$, que totalizaron 24.506. De ellos, 1701 murieron, una tasa de letalidad del $6,94 \%$. La comparación entre estos valores demuestra que el sobrepeso y la obesidad pueden influir en el pronóstico del paciente.

Se han reportado enfermedades cerebrovasculares en casos más graves de COVID19 (WANG et al., 2020). El mecanismo detrás del aumento de la gravedad para esta clase de pacientes es todavía incierto, aunque ya se sugiere que es similar al de las enfermedades cardiovasculares (PRANATA et al., 2020).

En cuanto a la relación entre las neoplasias y el COVID-19, se cree que los pacientes con cáncer son susceptibles al desarrollo de complicaciones debido al debilitamiento de su actividad inmunológica (ASOKAN; RABADIA; YANG, 2020; AL-QUTEIMAT; AMER, 2020). De hecho, un estudio retrospectivo de Zhang et al. (2020) mostró que, de 28 pacientes con cáncer evaluados, 8 murieron (tasa de mortalidad de 28,6\%), lo que indica un mal pronóstico para los pacientes con preexistencia de neoplasias.

La historia de fumar a su vez se especula como factor de riesgo importante para el desarrollo de condiciones severas de la enfermedad, puesto que fumar se asocia a la salud pulmonar empeorada (VARDAVAS; NIKITARA, 2020). Aunque solamente un caso de fumar se haya divulgado en los pacientes que murieron en nuestro análisis epidemiológico, hay los estudios que demuestran un riesgo creciente de severidad en pacientes con una historia de fumar. Alqahtani et al. (2020), en un metanálisis en el que participaron 139 fumadores actuales y 28 exfumadores diagnosticados con COVID-19, se encontró que 31 fumadores actuales (22,30\%) y 13 exfumadores $(46 \%)$ 
empeorado de sus condiciones clínicas. En el mismo estudio, murieron 5 fumadores actuales de los 13 que desarrollaron complicaciones (tasa de mortalidad del 38,5\%). Estas observaciones solo refuerzan que las personas que ya han fumado o fumado deben evitar la práctica en medio de la pandemia y siempre adoptar medidas preventivas.

Sobre la enfermedad de Alzheimer, la asociación entre la edad avanzada y la demencia, las características notables de la enfermedad, así como el posible deterioro del desarrollo neurocognitivo de estos individuos durante el aislamiento social en la pandemia, animan a la comunidad científica a evaluar la preexistencia del Alzheimer como factor de riesgo para COVID-19 (BRASIL, 2020b; FERINI-STRAMBI; SALSONE, 2020). En el caso de la mortalidad, Bianchetti et al. (2020) encontró que de 82 pacientes diagnosticados con COVID-19 y demencia, 51 (62,2\%) fallecieron, una tasa superior a la del $26,2 \%$ observada en pacientes que no tenían demencia, lo que señala que no solo la enfermedad de Alzheimer sino también otras demencias pueden estar asociadas con una afección grave de COVID-19.

El caso divulgado de un paciente etilista que murió sugiere hábito como factor de riesgo posible para la enfermedad. Esta asociación es apoyada por el conocimiento anterior que el abuso de alcohol puede comprometer la función hepática (EHRMANN; URBAN; DVORAN, 2019), que asustaría al paciente para que desarrolle complicaciones y, en consecuencia, un cuadro más grave de COVID-19.

La relación entre el puerperio y el COVID-19 no está tan bien establecida en comparación con el embarazo (AN et al., 2020). Mientras tanto, Tutiya et al. (2020) reportó dos casos de formación de microtrombo pulmonar en mujeres puerperales diagnosticadas con COVID-19. Además, An et al. (2020) reportaron dos casos de puérperas que presentaron dificultad respiratoria y empeoramiento de la afección después del parto. Estas observaciones refuerzan la necesidad de supervisar la condición de pacientes después de entrega hasta que haya estabilización.

Dengue y enfermedades pancreáticas que se han reportado en las muertes por COVID-19 en Amapá, sin embargo, no se han encontrado reportes en la literatura 
sobre la asociación de estas enfermedades y el desarrollo de complicaciones de la infección viral, aunque las enfermedades que comprometen la inmunidad del paciente tienden a aumentar las posibilidades de empeoramiento de las condiciones preexistentes (MARTINS-CHAVES; GOMES; GOMEZ, 2020).

\section{CONCLUSIÓN}

La preexistencia de comorbilidades $u$ otras afecciones que perjudican la respuesta inmune del cuerpo es un posible agravante de la afección infecciosa generada por el nuevo coronavirus, que puede llevar al paciente a la muerte. En el Estado de Amapá, gran parte de la población de pacientes diagnosticados con la enfermedad y fallecidos tenían antecedentes de factor de riesgo por COVID-19.

Las principales comorbilidades reportadas en pacientes diagnosticados con la enfermedad fueron diabetes, cardiopatía crónica, enfermedades respiratorias crónicas e hipertensión. La comorbilidad con mayor registro en casos de muerte fue la hipertensión.

La notificación de factores de riesgo en la tabla de defunción que no estaban registrados en la imagen de los pacientes diagnosticados, así como la inespecífica inherente a la condición de los pacientes monitoreados por los mecanismos de salud del Estado hizo un análisis más detallado sobre el grado de complicación y la tasa de letalidad generada por una determinada comorbilidad cuando se asoció con la infección por coronavirus.

Para una mayor exhaustividad y confiabilidad de los resultados de un estudio como este, es esencial que se obtengan datos suficientemente precisos y coherentes, para que el paso de los pacientes a través de la red de salud pueda ser analizado y luego incluido en la investigación científica, lo que permite comprender y enfrentar la pandemia actual y sus repercusiones en Amapá. 


\section{REFERENCIAS}

AGHAGOLI, G. et al. Cardiac involvement in COVID-19 patients: Risk factors, predictors, and complications: A review. J Card Sug., v. 35, p. 1302-1305, abr. 2020.

ALMEIDA, J. S. et al. Caracterização epidemiológica dos casos de covid-19 no maranhão: uma breve análise. Revista Prevenção de Infecção e Sáude, v. 6, mai. 2020.

AL-QUTEIMAT, O. M.; AMER, A. M. The Impact of the COVID-19 Pandemic on Cancer Patients. Am J Clin Oncol., abr. 2020. DOI: 10.1097/COC.0000000000000712.

ALQAHTANI, J. S. et al. Prevalence, Severity and Mortality associated with COPD and Smoking in patients with COVID-19: A Rapid Systematic Review and Meta-Analysis. PLoS One, v. 15, n. 5, e0233147, mai. 2020.

AN, P. et al. Postpartum exacerbation of antenatal COVID-19 pneumonia in 3 women. CMAJ, v. 192, n. 22, p. 603-606, jun. 2020.

ANGHEBEM, M. I.; REGO, F. G. M.; PICHETH, G. COVID-19 e Diabetes: a relação entre duas pandemias distintas. Revista Brasileira de Análises Clínicas. DOI: 10.21877/2448-3877.20200001. 2020.

ASKIN, L.; TANRIVERDI, O.; ASKIN, H. S. The Effect of Coronavirus Disease 2019 on Cardiovascular Diseases. Arq. Bras. Cardiol., São Paulo, v. 114, n. 5, p. 817-822, mai. 2020

ASOKAN, I.; RABADIA, S. V.; YANG, E. H. The COVID-19 Pandemic and its Impact on the Cardio-Oncology Population. Curr Oncol Rep., v. 22, n. 6, mai. 2020.

BIANCHETTI, A. et al. Clinical presentation of COVID-19 in dementia patients. J Nutr Health Aging, mai., 2020. DOI: 10.1007/s12603-020-1389-1. 
BORNSTEIN, S. R. et al. Practical recommendations for the management of diabetes in patients with COVID-19. The lancet Diabetes \& endocrinology, v. 9, n. 6, p. 546550, jun. 2020.

BRASIL. Plano de contingência DATASUS: Situação de crise provocada pelo novo coronavírus (covid-19). Versão 1.0, 2020a.

BRASIL. MINISTÉRIO DA SAÚDE. Alzheimer: o que é, causas, sintomas, tratamento, diagnóstico e prevenção. Disponível em:<https://saude.gov.br/saudede-a-z/alzheimer>. Acesso em: 31 jul. $2020 b$.

BRASIL. MINISTÉRIO DA SAÚDE. Painel de casos de doença pelo coronavírus 2019 (COVID-19) no Brasil pelo Ministério da Saúde. Versão 2.0. Disponível em:< https://covid.saude.gov.br/>. Acesso em: 10 de jul. de 2020c.

BUSSALINO, E. et al. Immunosuppressive therapy maintenance in a kidney transplant recipient SARS-CoV-2 pneumonia: a case report. Am J Transplant., abr. 2020. DOI: 10.1111/ajt.15920.

CAPARRO-GONZALEZ, R. A. COVID-19 in PregnantWomen and Neonates: A Systematic Review of the Literature with Quality Assessment of the Studies. Rev Esp Salud Pública., v. 94, abr. 2020.

CHENG, $Y$ et al. Kidney disease is associated with in-hospital death of patients with COVID-19. Kidney Internacional, v. 97, n. 5, p. 829-838, mar. 2020.

CHINAZZI, M. et al. The effect of travel restrictions on the spread of the 2019 novel coronavirus (COVID-19) outbreak. Science, v. 368, n. 6489, p. 395-400, mar. 2020.

COSTA, I. B. S. S. et al. O Coração e a COVID-19: O que o Cardiologista Precisa Saber. Arq. Bras. Cardiol., v. 114, n. 5, p. 805-816, mai. 2020a.

COSTA, F. F. et al. Metabolic syndrome and COVID-19: An update on the associated comorbidities and proposed therapies. Diabetes \& Metabolic Syndrome: Clinical Research \& Reviews, v. 14, n. 5, p. 809-814, 2020 b. 
DIAS, N. L. et al. Predição da propagação do SARS-CoV-2 no Estado do Amapá, Amazônia, Brasil, por modelagem matemática. Revista Científica Multidisciplinar Núcleo do Conhecimento, vol. 06, ano 05, ed. 05, p 73-95, mai., 2020.

DRIGGIN, E. et al. Cardiovascular Considerations for Patients, Health Care Workers, and Health Systems During the COVID-19 Pandemic. J Am Coll Cardiol., v. 75, n. 18, p. 2352-2371, mai. 2020.

ENGIN, A. B.; ENGIN, E. D.; ENGIN, A. Two important controversial risk factors in SARS-CoV-2 infection: obesity and smoking. Environmental Toxicology and Pharmacology, v. 78, mai. 2020. DOI: 10.1016/j.etap.2020.103411.

\section{ENGSTROM, E. et al. Recomendações para a organização da Atenção Primária à} Saúde no SUS no enfrentamento da Covid-19. Observatório Covid-19 Fiocruz, Nota técnica. p. 13, mai. 2020.

EHRMANN, J.; URBAN, O.; DVORAN, P. Alcohol-related liver diseases. Cent Eur J Public Health, v. 27, p. 10-14, dez. 2019.

FAN, C. et al. ACE2 Expression in Kidney and Testis May Cause Kidney and Testis Damage After 2019-nCoV Infection. MedRxiv, fev. 2020. DOI: 10.1101/2020.02.12.20022418.

FANG, L.; KARAKIULAKIS, G.; ROTH, M. Are patients with hypertension and diabetes mellitus at increased risk for COVID-19 infection?. The Lancet. Respiratory Medicine, v. 8, n. 4, mai. 2020.

FERINI-STRAMBI, L.; SALSONE, M. COVID-19 and neurological disorders: are neurodegenerative or neuroimmunological diseases more vulnerable?. J Neurol., jul. 2020. DOI: $10.1007 / \mathrm{s} 00415-020-10070-8$. 
FERRARI, F. COVID-19: Dados Atualizados e sua Relação Com o Sistema Cardiovascular. Arq. Bras. Cardiol., v. 114, n. 5, p. 823-826, mai. 2020. DOI: 10.36660/abc.20200215.

GAUTRET, P. et al. Hydroxychloroquine and azithromycin as a treatment of COVID19: results of an open-label non-randomized clinical trial. International journal of antimicrobial agents, mar. 2020. DOI: 10.1016/j.ijantimicag.2020.105949.

GIMENEZ, V. M. M. et al. Lungs as target of COVID-19 infection: Protective common molecular mechanisms of vitamin $D$ and melatonin as a new potential synergistic treatment. Life Sci., v. 354, ago. 2020. DOI: 10.1016/j.Ifs.2020.117808.

GUAN, W. et al. Clinical characteristics of coronavirus disease 2019 in China. New England journal of medicine, v. 382, n. 18, p. 1708-1720, fev. 2020.

HONG, H. et al. Clinical characteristics of novel coronavirus disease 2019 (COVID-19) in newborns, infants and children. Pediatrics and Neonatology, v. 61, n. 2, p. 131132, abr. 2020.

HRUSAK, O. et al. Flash survey on severe acute respiratory syndrome coronavirus-2 infections in paediatric patients on anticancer treatment. European Journal of Cancer, v. 132, p. 11-16, abr. 2020.

HUANG, C.; WANG, Y.; LI, X. Características clínicas de pacientes infectados com o novo coronavírus de 2019 em Wuhan, China. Lanceta, v. 395, p. 497-506, jan. 2020.

HUSSAIN, A. Obesity and mortality of COVID-19. Meta-analysis. Obes Res Clin Pract., jul. 2020. DOI: 10.1016/j.orcp.2020.07.002.

KHALIQ, O. P.; PHOSWA, W. N. Is Pregnancy a Risk Factor of COVID-19?. European Journal of Obstetrics \& Gynecology and Reproductive Biology, 2020. DOI: 10.1016/j.ejogrb.2020.06.058. 
IACOBELLIS, G. COVID-19 and diabetes: can DPP4 inhibition play a role?. Diabetes research and clinical practice, v. 162, abr. 2020. DOI: 10.1016/j.diabres.2020.108125.

LI, H. et al. Coronavirus disease 2019 (COVID-19): current status and future perspective. International journal of antimicrobial agents, v. 55, n. 5, mai. 2020.

LIU, H. et al. Why are pregnant women susceptible to COVID-19? An immunological Viewpoint. Journal of reproductive immunology, v. 139, jun. 2020.

LIMA, D. L. P. et al. COVID-19 no estado do Ceará, Brasil: comportamentos e crenças na chegada da pandemia. Ciênc. saúde coletiva, v. 25, n. 5, p. 1575-1586, mai. 2020.

LUCENA, T. M. C. et al. Mechanism of inflammatory response in associated comorbidities in COVID-19. Diabetes \& Metabolic Syndrome: Clinical Research \& Reviews, mai. 2020. DOI: 10.1016/j.dsx.2020.05.025.

MA, R. C. W.; HOLT, R. I. G. COVID-19 and diabetes. Diabetic Medicine, abr. 2020. DOI: $10.1111 / \mathrm{dme} .14300$.

MARTINS-CHAVES, R. R.; GOMES, C. C.; GOMEZ, R. S. Immunocompromised patients and coronavirus disease 2019: a review and recommendations for dental health care. Braz. res oral., São Paulo, v. 34, e048, mai. 2020.

MENDONÇA, F. D. Região Norte do Brasil e a pandemia de COVID-19: análise socioeconômica e epidemiológica. Journal Health NPEPS, v. 5, n. 1, 2020.

MOITINHO, M. S. et al. Lesão renal aguda pelo vírus SARS-COV-2 em pacientes com COVID-19: revisão integrativa. Rev. Bras. Enferm., Brasília, v. 73, n. 2, jul. 2020.

OLIVEIRA, A. C.; LUCAS, T. C.; IQUIAPAZA, R. A. What has the COVID-19 pandemic taught us about adopting preventive measures?. Texto \& Contexto Enfermagem, Florianópolis, v. 29, mai. 2020. DOI: 10.1590/1980-265x-tce-2020-0106. 
OLIVEIRA, W. K. et al. Como o Brasil pode deter a COVID-19. Epidemiol. Serv. Saude, Brasília, v. 9, n. 2, p. 1-8, abr. 2020.

OMS. ORGANIZAÇÃO MUDIAL DA SAÚDE. Painel da Doença de Coronavírus da OMS (COVID-19). Disponível em:< https://covid19.who.int/>. Acesso em: 10 de jul. de 2020.

OYELADE, T.; ALQAHTANI, J.; CANCIANI, G. Prognosis of COVID-19 in Patients with Liver and Kidney Diseases: An Early Systematic Review and Meta-Analysis. Trop. Med. Infect. Dis., v. 5, n. 80, mai. 2020.

PRANATA, R. et al. Impact of cerebrovascular and cardiovascular diseases on mortality and severity of COVID-19-systematic review, meta-analysis, and metaregression. J Stroke Cerebrovasc Dis., v. 29, n. 8,: mai. 2020.

PIRES, L. N.; CARVALHO, L.; XAVIER, L. L. COVID-19 e desigualdade: a distribuição dos fatores de risco no Brasil. Research Gate, abr. 2020. DOI: 10.13140/RG.2.2.27014.73282.

RAJKUMAR, R. P. Ayurveda and COVID-19: Where psychoneuroimmunology and the meaning response meet. Brain, behavior and immunity, v. 87, p. 8-9, jul. 2020.

RENU, K.; PRASANNA, P. L.; GOPALAKRISHNAN, A. V. Coronaviruses pathogenesis, comorbidities and multi-organ damage - A review. Life sci., v. 255, mai. 2020. DOI: 10.1016/j.Ifs.2020.117839.

RODRÍGUEZ-COLA, M. et al. Clinical features of coronavirus disease 2019 (COVID19 ) in a cohort of patients with disability due to spinal cord injury. Spinal Cord Series and Cases, v. 6, n. 39, mai. 2020.

RONCON, L. et al. Diabetic patients with COVID-19 infection are at higher risk of ICU admission and poor short-term outcome. Journal of Clinical Virology, v. 127, jun. 2020. DOI: 10.1016 / j.jcv.2020.104354. 
RUBIO-PÉREZ, I. et al. COVID-19: Conceptos clave para el cirujano. Cirugía Española, v. 98, n. 6, p. 310-319, jun./jul. 2020.

SIA, S. F. et al. Pathogenesis and transmission of SARS-CoV-2 in golden hamsters. Nature, mai. 2020. DOI: 10.1038/s41586-020-2342-5.

SILVA, A.W.C. et al. Caracterização clínica e epidemiologia de 1560 casos de COVID19 em Macapá/AP, extremo norte do Brasil. Research, Society and Development, v. 9, n.8, e150985499, jun. 2020.

STEFAN. N. et al. Obesity and impaired metabolic health in patients with COVID-19. Nat Rev Endocrinol., abr. 2020. DOI: 10.1038/s41574-020-0364-6.

TAY, M. Z. et al. The trinity of COVID-19: immunity, inflammation and intervention. Nat Rev Immunol, v. 20, p. 363-374, abr. 2020. DOI: 10.1038/s41577-020-0311-8.

TADIC, M. et al. COVID-19 and arterial hypertension: Hypothesis or evidence? J Clin Hypertens (Greenwich), jul. 2020. DOI:10.1111/jch.13925.

TRIPPELA, G. et al. COVID-19 in PregnantWomen and Neonates: A Systematic Review of the Literature with Quality Assessment of the Studies. Pathogens, v. 9, n. 6, jun. 2020.

TROYER, E. A.; KOHN, J. N.; HONG, S. Are we facing a crashing wave of neuropsychiatric sequelae of COVID-19? Neuropsychiatric symptoms and potential immunologic mechanisms. Brain, behavior and immunity, v. 87, p. 34-39, jul. 2020.

TUTIYA, C. T. et al. Possible formation of pulmonary microthrombi in the early puerperium of pregnant women critically ill with COVID-19: Two case reports. Case Rep Womens Health, v. 27, e00237, jun. 2020.

VARDAVAS, C. I.; NIKITARA, K. COVID-19 and smoking: A systematic review of the evidence. Tob Induc Dis., v. 18, mar. 2020. DOI: 10.18332/tid/119324. 
WANG, D. et al. Clinical Characteristics of 138 Hospitalized Patients With 2019 Novel Coronavirus-Infected Pneumonia in Wuhan, China. Journal of American Medical Asssociation, v. 323, n. 11, p. 1061-1069, fev. 2020.

YANG, J. et al. Prevalence of comorbidities and its effects in patients infected with SARS-CoV-2: a systematic review and meta-analysis. Int J Infect Dis., v. 94, p. 9195, mai. 2020.

YAO, H.; CHEN, J. H.; XU, Y. F. Patients with mental health disorders in the COVID19 epidemic. Lancet Psychiatry, v. 7, n. 4, mar. 2020.

ZHAI, P. et al. The epidemiology, diagnosis and treatment of COVID-19. International journal of antimicrobial agents, v. 55, n. 5, mai. 2020.

ZHANG, L. et al. Clinical characteristics of COVID-19-infected cancer patients: a retrospective case study in three hospitals within Wuhan, China. Ann Oncol., v. 31, n. 7, p 894-901, jul. 2020.

Enviado: Junio de 2021.

Aprobado: Junio de 2021. 